\title{
Galaxy properties from voids to clusters in the SDSS-DR4^
}

\author{
G. Sorrentino ${ }^{1,3,4}$, V. Antonuccio-Delogu ${ }^{2}$, and A. Rifatto ${ }^{1}$ \\ 1 INAF - Osservatorio Astronomico di Capodimonte, via Moiariello 16, 80131 Napoli, Italy \\ 2 INAF - Osservatorio Astrofisico di Catania, via S.Sofia 78, 95123 Catania, Italy \\ 3 Dipartimento di Fisica e Astronomia, Universitá di Catania, via S.Sofia 78, 95123 Catania, Italy \\ ${ }^{4}$ INAF - VST ceN, via Moiariello 16, 80131 Napoli, Italy
}

Received 9 June 2006 / Accepted 4 August 2006

\section{ABSTRACT}

\begin{abstract}
Aims. We investigate the environmental dependence of galaxy population properties in a complete volume-limited sample of 91566 galaxies in the redshift range $0.05 \leq z \leq 0.095$ and with $M_{r} \leq-20.0$ (that is $M^{*}+1.45$ ), selected from the Sloan Digital Sky Survey (SDSS) Data Release 4 (DR4). Our aim is to search for systematic variations in the properties of galaxies with the local galaxy density. In particular, we analyze how the $(u-r)$ color index and the morphological type of galaxies (the latter evaluated through the SDSS Eclass and FracDev parameters) are related to the environment and to the luminosity of galaxies, in order to find hints that can be related to the presence of a "void" galaxy population.

Methods. "Void" galaxies are identified through a highly selective criterion, which also takes redshift into account and allows us to exclude from the sample all the galaxies that are not really close to the center of underdense regions. We study the $(u-r)$ color distribution for galaxies in different luminosity bins, and we look for correlations of color with the environment, the luminosity, and the morphological type of the galaxies.

Results. We find that galaxies in underdense regions (voids) have lower luminosity $\left(M_{r}>-21\right)$ and are bluer than cluster galaxies. The transition from overdense to underdense environments is smooth, the fraction of late-type galaxies decreases, while the fraction of early-type galaxies increases smoothly from underdense to dense environments.

Conclusions. We do not find any sudden transition in the galaxy properties with density, which, according to a suggestion by Peebles (2001), should mark the transition to a population of "void" galaxies in $\Lambda$ CDM models. On the contrary, our results suggest a continuity of galaxy properties, from voids to clusters.
\end{abstract}

Key words. cosmology: large-scale structure of Universe - galaxies: fundamental parameters

\section{Introduction}

A self-consistent theory of galaxy formation and evolution within the gravitational collapse paradigm has yet to come, although quite significant advancements have been recently done (e.g. Silk 2004 for an up-to-date review). Such a theory should explain the observed properties of galaxy populations in very different environments, ranging from very dense clusters to large voids. However, the mere existence of galaxies within voids is a challenge for any gravitational bottom-up scenario of the Large Scale Structure (LSS) formation in the Universe, because in this scenario voids form out of the expansion of underdense $(\delta<0)$ initial density fluctuations. Theoretical models and numerical simulations (e.g. Olson \& Silk 1979; Bolejko et al. 2005) suggest that dark matter flows out of voids, and partly concentrates in some thin boundary shells. The latter, however, have not been detected, although there is clear evidence that the galaxy density profiles inside voids become steeper near their borders (Lindner et al. 1996; Hoyle \& Vogeley 2004; Goldberg et al. 2005). Observationally, there is evidence that voids are surrounded by superclusters of galaxies, as is vividly shown by recent analyses of the Las Campanas and SDSS surveys (Einasto et al. 2003a,b, 2005). It is interesing to note that the void models of Olson \& Silk (1979) and Hoyle et al. (2005b) are only concerned with the global dynamics of dark matter (DM): much less work has

^ Appendix is only available in electronic form at http: //www . aanda.org been devoted to understand the nonlinear collapse of DM halos and the formation of galaxies within voids. Dekel \& Silk (1986) suggested that voids could be populated by a population of faint dwarf galaxies, where star formation is suppressed by strong photoionization occurred at $z>6$ (Hoeft et al. 2005).

The search for galaxies in voids started with the discovery of the voids themselves (i.e., Lindner et al. 1996 for a full review in this field until 1996). Popescu et al. $(1996,1997)$ looked for emission-line galaxies in nearby voids, concluding that most of them are found close to the borders, and far from the deep internal regions. There have also been (failed) attempts at detecting a population of dwarf galaxies more homogeneously distributed within voids (Sabatini et al. 2003, 2005; Roberts et al. 2004), but the lack of an evident faint end of the dwarf galaxies luminosity function does not allow us to make firm quantitative predictions about their abundance.

Since the seminal papers by Grogin \& Geller $(1999,2000)$, some efforts have been dedicated to detect and to study the properties of bright galaxies in nearby voids. The availability of large samples of galaxies from surveys like the $2 \mathrm{dF}$ and the Sloan Digital Sky Survey (SDSS) has made possible more complete analysis of the dependence of galaxy properties on the environment. Croton et al. (2005) have shown that the luminosity function in voids tends to have a steeper slope at high luminosity, but not in the fainter range. The dependence of $(u-r)$ color distribution on the environment has also been recently studied by Balogh et al. (2004), who found that its shape is bimodal and can be well 
described by a linear combination of two Gaussian distributions for a very wide range of local densities. Surprisingly, they also find a small quantity of red, early-type galaxies even in the lowest density environments, thus suggesting that there is not a rigid morphological or color segregation with environment. However, the environmental dependence of galaxy properties is only detected for galaxies in the luminosity range $-20.4<M_{r}<-19.4$ (Tanaka et al. 2004): for galaxies brighter than $M_{r}=-20.4$ there are very little variations of their properties with the environment. Peebles (2001) suggested that galaxies lying within very underdense regions are representative of a distinct population. In this case, we should observe a transition in one or more galaxy properties moving from clusters to voids.

In order to verify the previous hypothesis, we will investigate the variation of the color distribution, morphology, and luminosity with the local density. This kind of analysis has been made possible by the availability of a large sample of galaxies extracted from the SDSS - Data Release 4 (SDSS-DR4: York et al. 2000; Stoughton et al. 2002; Abazajian et al. 2004). Using this huge data sample we confirm some previous results (e.g. Rojas et al. 2004), and we are able to extract statistically significant subsamples for different relative underdensities. We will study the correlation between $(u-r)$ colors and the environment for bright and faint galaxies, also looking for sudden changes which should mark the emergence of a distinct void galaxy populations, as suggested by Peebles (2001). We will use a criterion to estimate the density which is different from the $\Sigma_{5}$ criterion used by, e.g., Tanaka et al. (2004); on the contrary, it is more similar to the one adopted by Rojas et al. (2004), because we want to be sure that galaxies in voids are correctly identified and included in the sample.

The plan of the paper is as follows: after the description of the galaxy sample (Sect. 2), in Sect. 3 we will discuss the adopted criteria to construct our subsamples. In Sect. 4 we will present our results concerning the $(u-r)$ color distribution, and its dependence on the local density and luminosity. In Sect. 5 we will analize the results of the previous section taking also into account the relationship between the $(u-r)$ color distribution and the morphological type of galaxies, then discussing the relationship with the morphology-density relation (Dressler 1980). Finally, in Sects. 6 and 7 we will present a general discussion and our conclusions.

Throughout, we adopt standard present day values of the cosmological parameters to compute comoving distances from redshift: a density parameter $\Omega_{\mathrm{m}}=0.3$, a cosmological constant $\Omega_{\Lambda}=0.7$, and a Hubble's constant value $H_{0}=75 \mathrm{~km} \mathrm{~s}^{-1} \mathrm{Mpc}^{-1}$.

\section{SDSS - DR4}

The SDSS (York et al. 2000; Abazajian et al. 2004, 2005; Adelman-McCarthy 2005) is a photometric and spectroscopic survey, which will map about one quarter of the entire sky outside the Galactic plane, and will collect spectra of about $10^{6}$ galaxies, $10^{5}$ quasars, 30000 stars and 30000 serendipity targets.

Photometry is available in $u^{\prime}, g^{\prime}, r^{\prime}, i^{\prime}$ and $z^{\prime}$ bands (Fukugita et al. 1996; Gunn et al. 1998), while the spectroscopic data are obtained with a pair of multi-fiber spectrographs. Each fiber has a diameter of $0.2 \mathrm{~mm}$ ( $3^{\prime \prime}$ on the sky), and adjacent fibers cannot be located more closely than $55^{\prime \prime}$ on the sky $(\sim 110 \mathrm{kpc}$ at $z=0.1$ with $H_{0}=75 \mathrm{~km} \mathrm{~s}^{-1} \mathrm{Mpc}^{-1}$ ) during the same observation. In order to optimize the placement of fibers on individual plates, and as the placement of plates relative to each other, a tiling method has been developed which allows a sampling rate
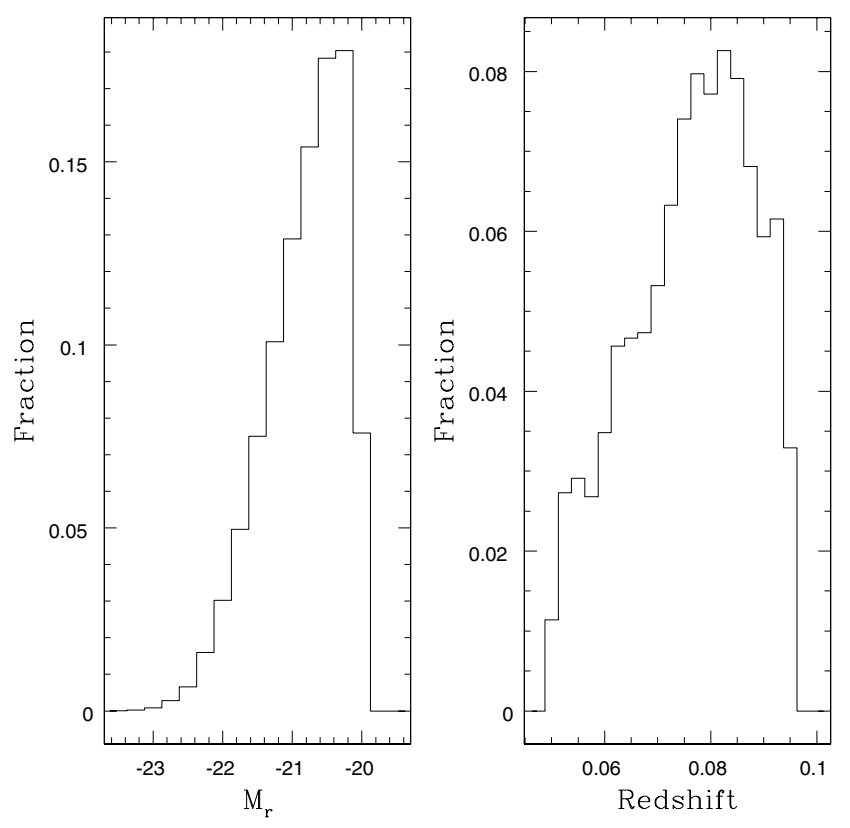

Fig. 1. The redshift (right), and $r$-band absolute magnitude distributions (left), for the 91566 galaxies in our sample. The absolute magnitude corresponding to the upper redshift limit $(z=0.095)$ is $M_{r}=-20.0$.

of more than $92 \%$ for all targets. For details see the SDSS web site (www.sdss.org/dr4/algorithms/tiling.html).

The spectroscopic SDSS-DR4 catalog contains about 673280 galaxies and covers an area of 4783 square degrees. The spectra cover the spectral range $3800<\lambda<9200 \AA$, with a resolution of $1800<\lambda / \Delta \lambda<2100$, and give a rms redshift accuracy of $30 \mathrm{~km} \mathrm{~s}^{-1}$, to an apparent magnitude limit (Petrosian magnitude) of $r^{\prime}=17.77$.

Data have been obtained from the SDSS database (http://www.sdss.org/DR4) using the CasJobs facility (http://casjobs.sdss.org/casjobs/).

\section{Sample selection}

In this paper, we will take into account a complete volumelimited sample of galaxies in the redshift range $0.05 \leq z \leq$ 0.095 , brighter than $M_{r}=-20.0$, that is $M_{r}^{*}+1.45$, with $M_{r}^{*}=-21.45$. The lower redshift limit is chosen with the aim of minimizing the aperture bias (e.g. Gómez et al. 2003) caused by the presence of large nearby galaxies, while the upper limit is aimed at obtaining a high level of completeness, estimated through Schmidt's $V / V_{\max }$ test.

Our initial sample contains 91566 galaxies. For each galaxy, we compute its $r$-band absolute magnitude, dereddened and $\mathrm{K}$ corrected as suggested in Blanton et al. (2003). The distributions of $z$ and $M_{r}$ are given in Fig. 1.

Galaxies in our sample with no detectable emission lines in their spectra are classified as Passive Galaxies (PGs): for these galaxies, the morphological type should be earlier than Sa. Galaxies with one or more emission lines having $I_{\lambda} / \sigma_{I_{\lambda}}>2$, where $I_{\lambda}$ is the emission line flux and $\sigma_{I_{\lambda}}$ its uncertainty, are classified as star forming galaxies (SFGs), according to the criteria adopted by Kewley et al. (2001): for these galaxies, the morphological type should be later than S0/Sa. In order to avoid all the ambiguous cases in the AGN/SFG classification, 
Table 1. Numbers of neighbours and the related percentile. A percentile is defined as the value of a given scalar quantity characterizing the fraction of the distribution that is equal or smaller than that value.

\begin{tabular}{rr}
\hline \hline$N_{\text {neigh }}$ & Percentile \\
\hline 0 & $1 \mathrm{st}$ \\
2 & 5 th \\
4 & 10 th \\
9 & 25 th \\
18 & 50 th \\
35 & 75 th \\
59 & 90 th \\
79 & 95 th \\
127 & 99 th \\
\hline
\end{tabular}

we removed those sources whose line ratios fall close to the border line of the diagnostic diagrams. This was done by keeping only those sources for which part of the error bar associated to the logarithm of the line-ratios lie within the theoretical uncertainty of the model $\left(\sigma_{\bmod }=0.1 \mathrm{dex}\right)$ in both $x$ and $y$ directions.

Moreover, we adopted another criterion to separate the galaxies in early- and late-type, according to their morphological type defined by the two parameters ECLASS and FRACDEV provided by the SDSS. FRACDEV is a photometric parameter providing the weight of a deVaucouleurs component in best composite exponential+deVaucouleurs models, and ECLASS is a spectroscopic parameter giving the spectral type from a principal component analysis. Early-type galaxies $(\mathrm{E}+\mathrm{S} 0)$ were selected following the criteria adopted by Bernardi et al. (2005): FRACDEV $(r)>0.8$ and ECLASS $<0$. Late-type galaxies ( Sa and later) were selected when either ECLASS $\geq 0$ or FRACDEV $(r)<0.5$. In this way we exclude from our analysis all the galaxies for which an unambiguous classification is not possible, because their ECLASS and FRACDEV parameters are out of the previous defined ranges.

In order to investigate a possible dependence of galaxy colors on the environment, for each galaxy we compute the number of neighbours within a fixed radius $D_{\max }=5 \mathrm{Mpc}$, the distribution and the related percentile (Table 1). We adopted this particular distance because it was used in previous works aimed at studying galaxies in very underdense regions (voids) (Grogin \& Geller 1999; Hoyle \& Vogeley 2004). The use of the percentile in our analysis allows us to compare different environments and to characterize the difference between over- and underdense regions without defining an environment a priori.

A galaxy $j$ is considered as a neighbour of a galaxy $i$ if:

$$
\begin{aligned}
& \text { - } D_{i j} \leq D_{\max } \\
& \text { - } c\left|z_{i}-z_{j}\right| \leq 1000 \mathrm{~km} \mathrm{~s}^{-1}
\end{aligned}
$$

where $D_{i j}$ is the projected distance between the two galaxies, and $\left|z_{i}-z_{j}\right|$ is their redshift difference. $D_{i j}$ is computed from the angular separation $\theta_{i j}$ and the redshift $z_{i}$; the limit $c\left|z_{i}-z_{j}\right| \leq$ $1000 \mathrm{~km} \mathrm{~s}^{-1}$ is the value usually adopted to select cluster or galaxy group members in the velocity space (Fadda et al. 1996; Wilman et al. 2005). Throughout, we intend as local galaxy density the number of neighbours brighter than $M_{r}=-20.00$ and within $5 \mathrm{Mpc}$.

In Appendix A we explore the effect of varying our definition of local galaxy density on scales $2.5 \mathrm{Mpc}$ to $10 \mathrm{Mpc}$. We find that our conclusions remain unchanged.

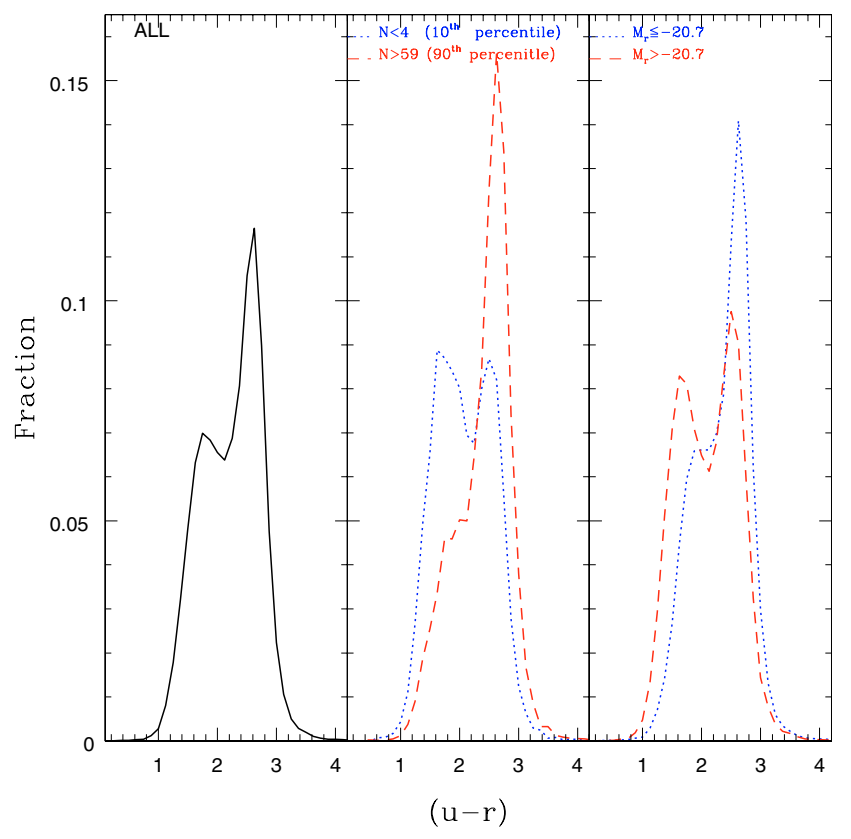

Fig. 2. Left panel: the $(u-r)$ color distribution for all the galaxies in our sample. Central panel: the $(u-r)$ color distribution for galaxies having less than 4 neighbours (corresponding to 10th percentile) compared with the distribution of galaxies having more than 59 neighbours (corresponding to 90th percentile) within $5 \mathrm{Mpc}$. Right panel: the $(u-r)$ color distribution for galaxies brighter and fainter than $M_{r}=-20.7$.

\section{4. $(u-r)$ color distribution: dependence on environment and luminosity}

It is well known that the environment can affect the colors of galaxies, and that galaxies in low density environment are generally bluer than galaxies in clusters (Grogin \& Geller 1999). However, we can not relate the color of galaxies to their environment because the $(u-r)$ color does not correlate with the local density in a single way. Then, because the luminosity function of bright galaxies decreases steeply with increasing luminosity, we investigated whether the differences between cluster and void galaxies are also a function of their luminosity, as suggested by Rojas et al. (2004). In Fig. 2, we analize the $(u-r)$ color distribution for all the galaxies in our sample (left panel), for galaxies in different environments (central panel), and for two different ranges of luminosity (right panel). To this last aim, we adopted the median value of the luminosity distribution to define two different ranges of luminosities: $M_{r}$ fainter and brighter than $M_{r}=-20.7$. Galaxies in underdense environments (defined as those having $N<4$ neighbours within $5 \mathrm{Mpc}$, where $N=4$ corresponds to the 10 th percentile) and galaxies fainter than $M_{r}=-20.7$ have a similar, bimodal, color distribution. The second peak is redder and coinciding with the peak of the color distribution for galaxies in dense environments $(N>59$ neighbours, corresponding to the 90th percentile) and brighter than $M_{r}=-20.7$. From this first analysis, it is evident that, in general, galaxies in underdense environments are bluer and fainter than galaxies in dense environments.

In Fig. 3, differences between over- and underdense regions, as characterized by the number of neighbours, are increasing from left to right. For instance, in the rightmost panels, the distributions are referred to galaxies having more than 127 or less than 1 neighbours within $5 \mathrm{Mpc}$, corresponding to the 1 st and 


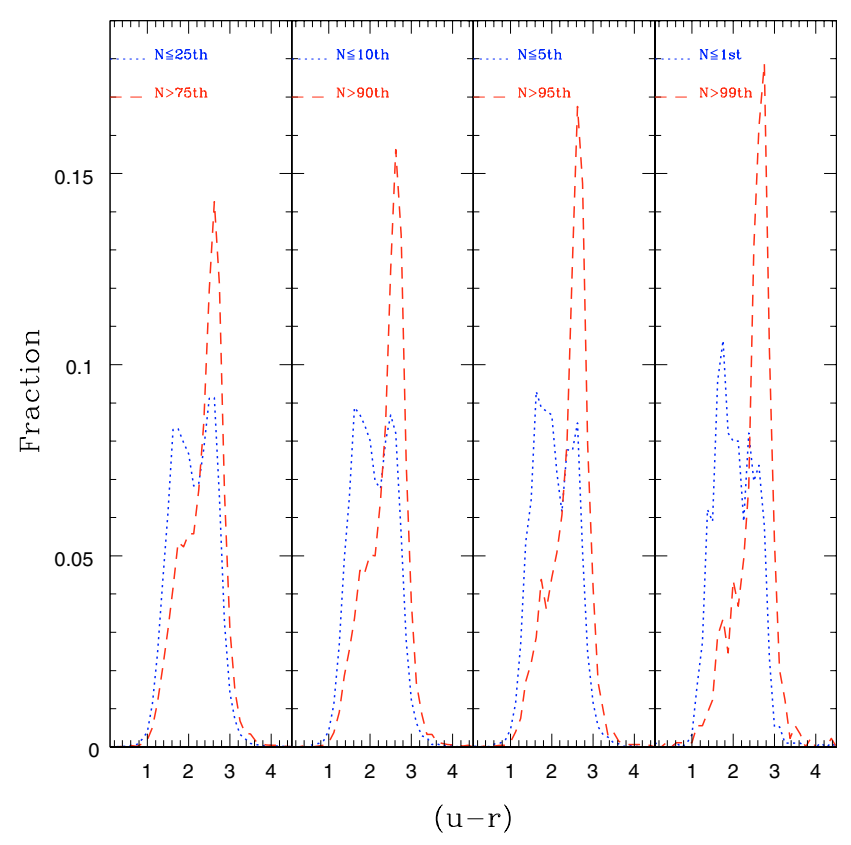

Fig. 3. $(u-r)$ color distribution for galaxies in different environments.

99th percentiles of the global distribution, respectively. In this way we are able to compare environments that are more and more extreme. These plots confirm that in underdense regions galaxies tend to be bluer than cluster galaxies, and the transition from over- to under-dense regions is smooth. In Fig. 5, the galaxy populations for different environment are compared, using progressively fainter galaxies, from left to right. Galaxies in underdense environment $\left(N_{\text {neigh }} \leq 25\right.$ th or $N_{\text {neigh }} \leq 5$ th $)$ are bluer at fainter luminosities and the transition is smooth: this result is confirmed by a KS test and it is also in accordance with Rojas et al. (2004). Then, we conclude that both luminosity and density act to discriminate galaxies in underdense environments from galaxies in the field or in clusters.

In the following, we will make these statements more quantitative.

In order to quantify the difference between over- and underdense regions, we compute a $\Delta$ median value of $(u-r)$, defined as the difference of the median value $(u-r)$ between the distributions of two opposite percentile. We define as opposite percentile the value $1-p$, being $p$ the value of the percentile. For example, for the 20th percentile the opposite percentile is the 80th so that, for this example, $\Delta$ median $_{20 \text { th }}$ will be:

smedian $_{20 \text { th }}=(u-r)_{N<20 \text { th }}-(u-r)_{N>80 \text { th }}$

where $(u-r)$ is the median value of the distribution.

The plot in Fig. 4 shows a clear trend in the variation of the previous quantity with the color. It is interesting to observe that the variation is a smooth function of the difference in percentiles: we do not observe a very steep change of the slope for small values of the percentile, i.e., for very different environments. This result is a further hint at the fact that there is not a "void galaxy population" with radically distinct properties, as suggested by Peebles (2001) for a standard CDM model.

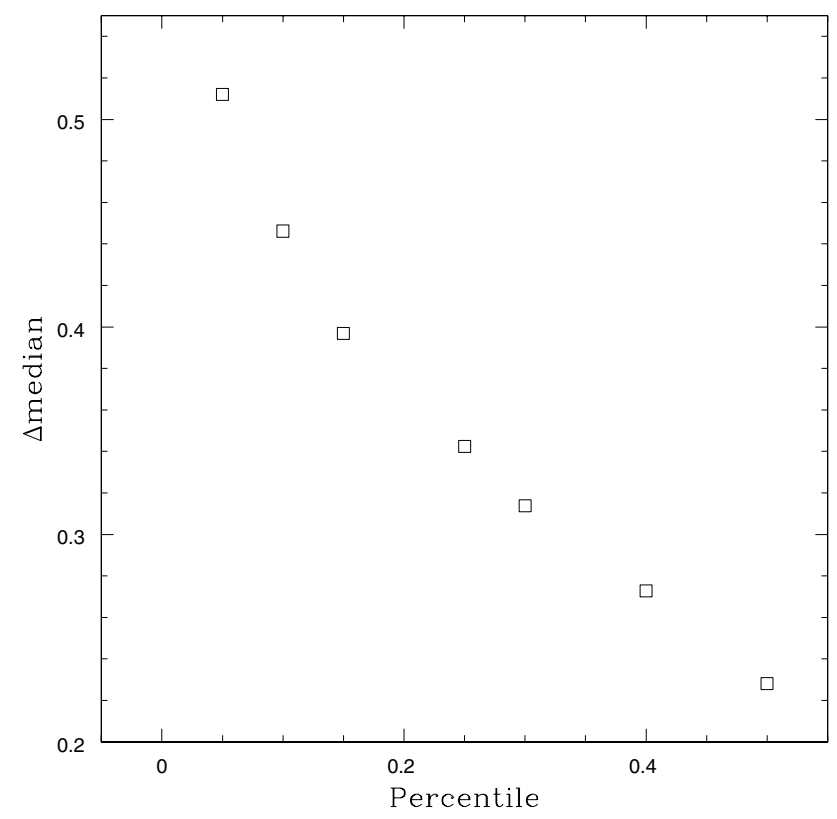

Fig. 4. The trend of $\Delta$ median, defined in the text, as a function of the percentile.

\section{5. $(u-r)$ color distribution: dependence on morphology}

In the previous section, we studied the dependence of the $(u-r)$ color distribution for the galaxies on both the environment and the luminosity, without to consider any dependence on their morphological type. But it is well known that the morphological type of galaxies correlates with density (Dressler 1980) and color (Strateva et al. 2001), as well as there is also a correlation between density, luminosity and color (Hogg et al. 2003). We also found that galaxies in underdense environments are bluer than galaxies in dense environment, in accordance with Baldry et al. (2004), but it is unclear if this is an induced effect driven by some other relation, e.g. the morphology-density relation (Dressler 1980). Because the value $(u-r)=2.22$ can be used to separate early- from late-type galaxies (Strateva et al. 2001) and because a clear bimodality is observed in the $(u-r)$ color distribution, in this section we will investigate the existence of a possible correlation between the findings in Sect. 4 and the morphology. In particular, in order to relate the morphology to the environment, we compare the $(u-r)$ color distribution for early- and late-type galaxies with the color distribution of the total sample of galaxies.

From Fig. 6 we see that the bimodal shape of the $(u-r)$ color distribution is a function of both the environment and the morphological type, becoming redder with increasing the density of the environments, as well as the fraction of early-type galaxies: in poor systems (i.e. $0 \leq N<4$ ) the fractions of earlyand late-type galaxies are similar; with increasing the number of neighbours the fraction of early-type galaxies also increases and the bimodality almost disappears (i.e. $59 \leq N<127$ ). The numbers $N$ of galaxies used in Fig. 6 are not casual, but they rather correspond to the percentiles of the total distribution (Table 1).

In the previous section it has also been observed that the bimodal $(u-r)$ color distribution depends on both the environment and the luminosity. In fact, the fraction of blue galaxies 


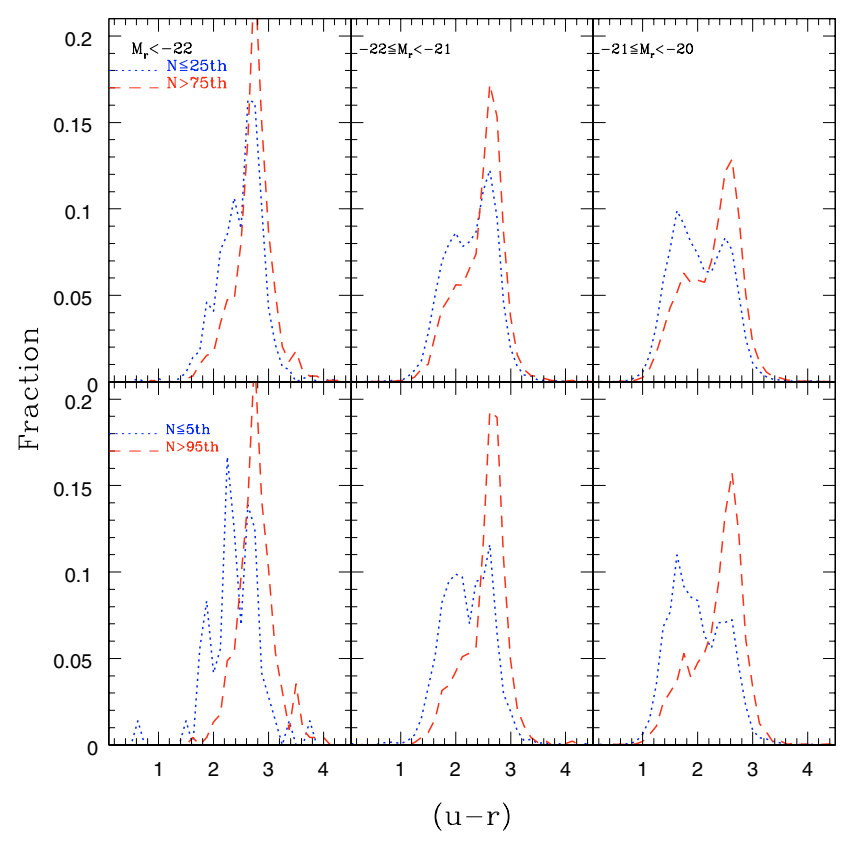

Fig. 5. Top panels: comparison of the color distribution for galaxies having a number of neighbours lesser than the 25 th percentile with the color distribution for galaxies having a number of neighbours larger than the 75th percentile. Bottom panels: comparison of the color distribution for galaxies having a number of neighbours lower than the 5 th percentile with the color distribution for galaxies having a number of neighbours greater than the 95 th percentile. In both panels, the luminosity decreases from left to right.

Table 2. Number of galaxies in the plots of Fig. 6.

\begin{tabular}{crrr}
\hline \hline & All & Early (\%) & Late (\%) \\
\hline $0 \leq N<4$ & 7205 & 31.3 & 31.7 \\
$4 \leq N<7$ & 8402 & 36.2 & 28.4 \\
$7 \leq N<11$ & 17490 & 38.0 & 26.7 \\
$11 \leq N<18$ & 17393 & 41.1 & 24.1 \\
$18 \leq N<30$ & 18608 & 45.2 & 21.1 \\
$30 \leq N<41$ & 9595 & 46.8 & 18.8 \\
$41 \leq N<59$ & 9215 & 50.4 & 16.6 \\
$59 \leq N<127$ & 8470 & 55.1 & 14.0 \\
\hline
\end{tabular}

decreases becoming more and more red with increasing of both the environmental density and the luminosity (Fig. 5), in accordance with Baldry et al. (2004) and Menci et al. (2005). Then in order to relate this result to the morphological type, we plot the $(u-r)$ color distribution for different ranges of luminosities and morphologies (Fig. 7). We find that the fraction of earlytype galaxies decreases, while the fraction of late-type galaxies increases in samples which are over fainter, then probing the environmental dependence on the luminosity of the $(u-r)$ color distribution for early- and late-type galaxies.

Finally, we condider the $(u-r)$ color distribution for galaxies having different morphological type and in different bins of magnitudes. Galaxies with $-21 \leq M_{r}<-20$ have a similar fraction of early $(36.0 \%)$ and late $(29.8 \%)$ type galaxies; for $-22 \leq M_{r}<-21$ these fractions are $55.1 \%$ (early) and $9.3 \%$ (late), while for $M_{r}<-22$ the fraction of late-type galaxies is negligible $(1.5 \%)$ compared to the fraction of early-type $(75.3 \%)$. In general, we have a similar trend (Fig. 7): the fraction

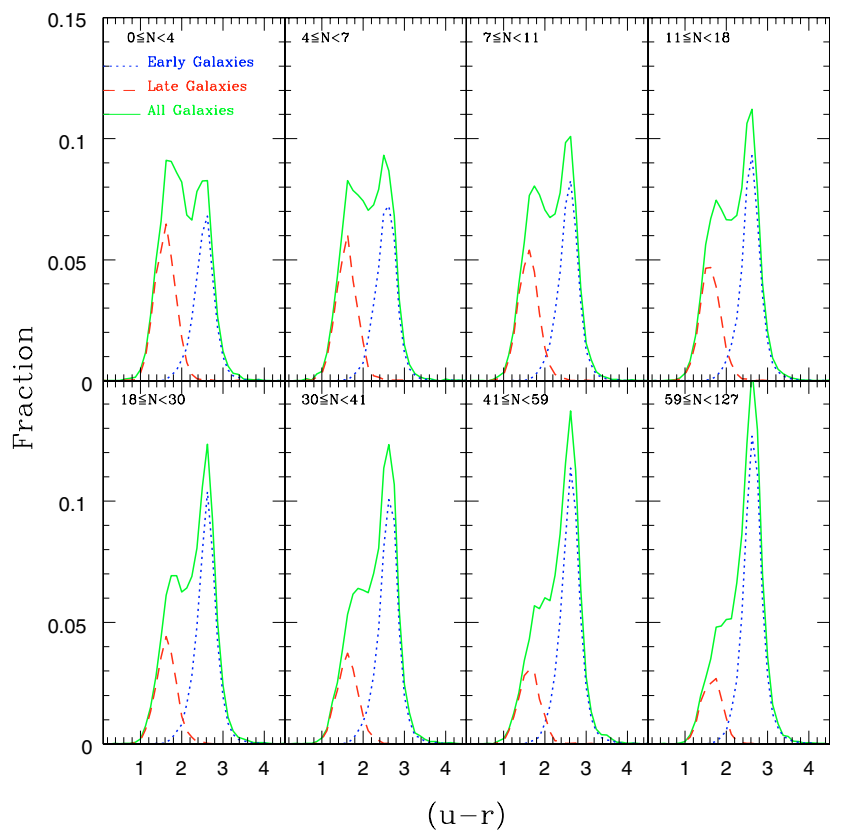

Fig. 6. $(u-r)$ color distribution for different environment ( $N$ is the number of neighbours within $5 \mathrm{Mpc}$ ) and for different morphological types.

of late-type galaxies decreases with increasing of both the environmental density and luminosity, while the fraction of earlytype galaxies increases, then confirming the previous findings concerning the environmental dependence on the luminosity of the $(u-r)$ color distribution for early- and late-type galaxies. Moreover, the color distributions for early-type/PGs and latetype/SFGs galaxies defined as described in Sect. 3, are similar (Fig. 8), so that we can relate the environmental properties of late-type galaxies not only to the morphology-density relation (Dressler 1980), but also to their star-forming activity.

\section{Discussion}

By definition, there are few galaxies in voids, and only the availability of large samples can allow to build statistically significant catalogues of galaxies in such underdense regions. These conditions are met by the sample we use in this paper, extracted from the SDSS-DR4.

Studying the galaxy properties in extremely underdense environments is an interesting test for cosmological and galaxy formation models. In standard CDM models, underdense regions should be populated with sub-L* galaxies (Dekel \& Silk 1986). Many of these "void dwarfs" were successfully identified in previous surveys. For example Lindner et al. $(1995,1996)$ found that their density decreases towards the center of the voids themselves. Recent surveys like the SDSS show the presence of both isolated galaxies and filaments thereof within voids (Einasto et al. 2003b), a circumstance which is also the result of numerical simulations (Antonuccio-Delogu et al. 2002; Gottlöber et al. 2003). Because these coherent structures are difficult to identify in galaxy surveys, recently many effort have been carried out to detect filaments (Pimbblet 2005).

Peebles (2001) notices that the existence of a particular class of void-galaxies marked by a discontinuity in the observed properties of galaxy populations, from over- to underdense regions, would be a distinctive feature of galaxy formation in CDM 


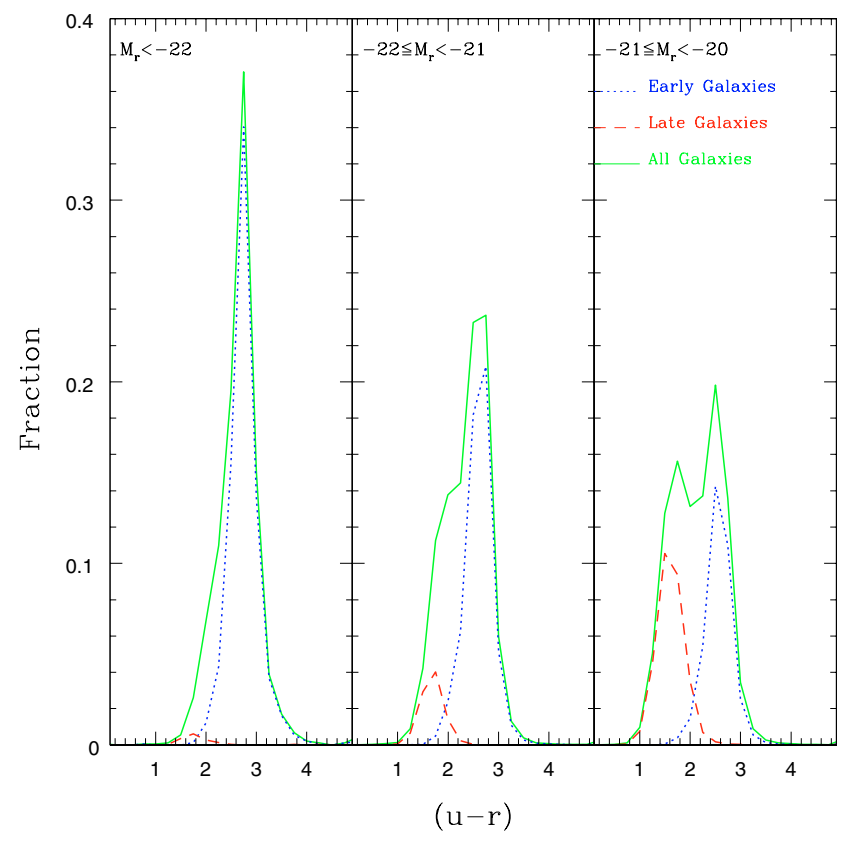

Fig. 7. $(u-r)$ color distribution for galaxies having different luminosities and different morphological types.

Table 3. Number of galaxies in the plots of Fig. 7

\begin{tabular}{crrr}
\hline \hline & All & Early (\%) & Late (\%) \\
\hline$M_{r}<-22$ & 3617 & 75.3 & 1.5 \\
$-22 \leq M_{r}<-21$ & 27815 & 55.1 & 9.3 \\
$-21 \leq M_{r} \leq-20$ & 60134 & 36.0 & 29.8 \\
\hline
\end{tabular}

models. On the contrary, our results (e.g., Fig. 5) point out a smooth transition in colors and other properties with average density. This result is also confirmed when we consider the difference between truly void-galaxies and "wall" galaxies, then suggesting that does not exist a "pure" void-galaxy population, distinguished from the average galaxy population.

However, the only study of galaxy colors as a function of the environment gives a partial view. In fact, the color of galaxies in underdense environments has a different distribution from that of galaxies in dense environment (red), as shown in Fig. 2. On the contrary, in underdense environments, the distribution of the galaxy populations without taking into account the morphological type is not equally skewed towards blue colors, confirming the findings by Balogh et al. (2004). Galaxies become bluer only if we consider a very underdense environment.

When we consider galaxies fainter than $M_{r}=-22.0$, their color becomes more and more blue in environments that are more and more underdense $\left(N_{\text {neigh }}<25\right.$ th, $N_{\text {neigh }}<10$ th, $N_{\text {neigh }}<5$ th, $N_{\text {neigh }}<1$ st). A similar trend (shifted to the red) is observed for galaxies in environments more and more dense $\left(N_{\text {neigh }}>75\right.$ th, $N_{\text {neigh }}>90$ th, $N_{\text {neigh }}>95$ th, $N_{\text {neigh }}>99$ th $)$, (Fig. 3). This trend becomes more and more evident when we consider galaxies more and more faint. We find that in dense environments, galaxies fainter than $M_{r}=-21$ are redder than galaxies in underdense environments (Fig. 5), confirming that the color distribution of galaxies is also strongly dependent on the absolute magnitude. Then, we can assert that the $(u-r)$ color

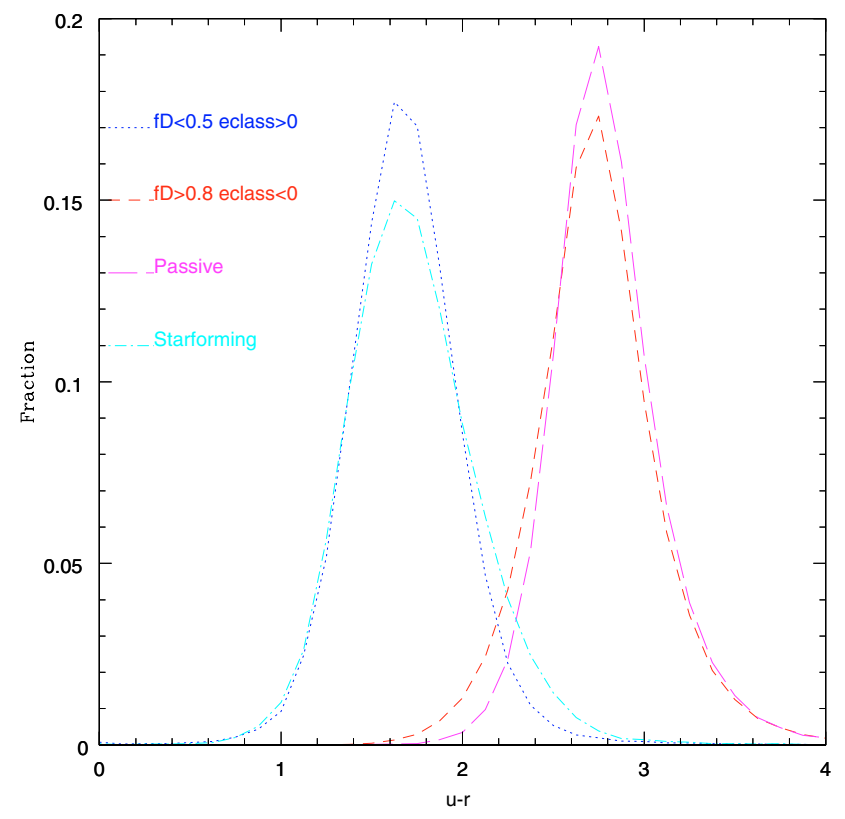

Fig. 8. The $(u-r)$ color distribution for early- and late-type galaxies spectroscopically selected by using the presence of emission lines in the spectra and the FracDev $v_{r}$ and Eclass parameters as in Bernardi et al. (2005). The two distributions are in good agreement.

distribution of galaxies is both related to the environment and to the luminosity of the galaxies.

These results are in agreement with Croton et al. (2005) and Einasto et al. (2005) who showed that luminosity function parameters and brightest luminosities of galaxies are smooth functions of the density of the environment.

When we take into account the morphological type of galaxies, we find that the fraction of late-type is bluer and fainter than the fraction of early-type galaxies, and that their fraction decreases from low $(0 \leq N<4)$ to dense environment $(59 \leq N<127)$, while the early-type galaxies fraction increases (Fig. 6). In particular, in the range of luminosities brighter than $M_{r}=-22.0$, we have an excess of early-type $(75.3 \%)$ respect to late-type $(1.5 \%)$ galaxies, while for low luminosities $\left(M_{r}>-21\right)$ the fraction of late-type galaxies increases $(29.8 \%)$ compared to fraction of early-type (36.0\%) (Fig. 7 and Table 3). This result is in accordance with the morphology-density relation (Dressler 1980), confirming that, on average, early-type galaxies are redder, brighter, and in denser environments than late-type galaxies.

It is important to notice that the $(u-r)$ color distributions for early-type and late-type galaxies, defined through the SDSS Eclass and FracDev parameters, are similar to the color distribution for PGs and SFGs, respectively (Fig. 8). Then, the environmental properties of late-type galaxies can be related to the morphology-density relation (Dressler 1980) not only as a consequence of their morphological type, but also for their starforming activity. This latter point is not completely unexpected. In fact, in the sample of galaxies used by Grogin \& Geller (2000, 1999), there are a lot of galaxies in voids that are found in pairs or small groups. Thus, enhanced stellar formation due to tidal effects is likely to be detected among void galaxies.

In a recent paper, Patiri et al. (2006) analysed the properties of voids galaxies in the SDSS-DR4, finding some results similar to ours, i.e., a bimodal distribution of the $(u-r)$ color index and 
a general trend for galaxies in voids to be bluer than in clusters: this latter result is strongly dependent on the absolute magnitude, as we demonstrate in this paper. In particular, they also found no difference between void and field galaxies if only galaxies in a restricted absolute magnitude range are analysed.

\section{Conclusions}

In this paper we analyzed the environmental dependence of galaxy population properties, looking for those features which could hint at the presence of a "void" galaxy population. The photometric properties we used in our analysis can be regarded as complementary respect to those used by other authors (Rojas et al. 2004; Hoyle et al. 2005a). In particular, our density estimator allows us to exploit redshift information, then finding galaxies in very underdense regions.

We can summarize our findings in a few points:

1. Luminosity is a sensitive parameter to characterize the environmental properties of galaxies.

2. On average, a very underdense environment $\left(N_{\text {neigh }} \leq 4\right)$ is populated by galaxies which are bluer than galaxies that are in a very dense environments, $\left(N_{\text {neigh }}>79\right.$, see Fig. 2$)$.

3. On average, faint galaxies $\left(M_{r}>-21.0\right)$ are bluer in very underdense environments than in dense environments and the transition from blue to red, moving from under- to overdense environments, is more pronounced for faint galaxies $\left(M_{r}>-21.0\right.$, Fig. 5).

4. Although the transition from voids to clusters is more pronounced for faint galaxies, it does not seem to be a discontinuity (Fig. 4).

5. Changes in the $(u-r)$ color distribution are related to the environment, to the luminosity, and to the morphology of the galaxies. In fact, on average, fainter galaxies $\left(M_{r}>-21.0\right)$ are bluer, late-type and in underdense regions (voids) than brighter galaxies $\left(M_{r}<-21.0\right)$, which are redder, early-type and in overdense regions (clusters).

6. Galaxies classified as early-type/late-type and PGs/SFGs, according to their photometric/spectroscopic parameters and to their class of star-forming activity, have a similar $(u-r)$ color distribution (Fig. 8). Then, the environmental properties of late-type galaxies can be related to the morphologydensity relation (Dressler 1980) not only as a consequence of their morphological type, but also as a consequence of their star-forming activity.

7. We don't find any sudden transition in the properties of "void" galaxies, with respect to cluster galaxies, as suggested by Peebles (2001). On the contrary, our results show a continuity in the properties of the galaxies, from voids to clusters.

Acknowledgements. We are grateful to Chris Scott Kay and C. Haines for having carefully read the manuscript and, C. Haines for the useful conversations and comments.

We wish to thank the referee, Jaan Einasto, for the constructive comments which improved the content of the paper.

G.S. and A.R. thank the EC and the MIUR for having partially supported this work (EC contracts HPRN-CT-2002-00316 - SISCO network, and MIURCOFIN-2004 n.2004020323_001).

V.A. gratefully acknowledges the support from the European Commission (Marie Curie "Transfer of Knowledge" Project COSMOCT, contract MC-TOK002995.)

Funding for the SDSS and SDSS-II has been provided by the Alfred P. Sloan Foundation, the Participating Institutions, the National Science Foundation, the US Department of Energy, the National Aeronautics and Space Administration, the Japanese Monbukagakusho, the Max Planck Society, and the Higher Education Funding Council for England. The SDSS Web Site is http://www. sdss.org/.
The SDSS is managed by the Astrophysical Research Consortium for the Participating Institutions. The Participating Institutions are the American Museum of Natural History, Astrophysical Institute Potsdam, University of Basel, Cambridge University, Case Western Reserve University, University of Chicago, Drexel University, Fermilab, the Institute for Advanced Study, the Japan Participation Group, Johns Hopkins University, the Joint Institute for Nuclear Astrophysics, the Kavli Institute for Particle Astrophysics and Cosmology, the Korean Scientist Group, the Chinese Academy of Sciences (LAMOST), Los Alamos National Laboratory, the Max-Planck-Institute for Astronomy (MPA), the Max-Planck-Institute for Astrophysics (MPIA), New Mexico State University, Ohio State University, University of Pittsburgh, University of Portsmouth, Princeton University, the United States Naval Observatory, and the University of Washington.

\section{References}

Abazajian, K., Adelman-McCarthy, J. K., Agüeros, M. A., et al. 2004, AJ, 128, 502

Abazajian, K., Adelman-McCarthy, J. K., Agüeros, M. A., et al. 2005, AJ, 129, 1755

Adelman-McCarthy, J. K. 2005, ArXiv Astrophysics e-prints

Antonuccio-Delogu, V., Becciani, U., van Kampen, E., et al. 2002, MNRAS, 332, 7

Baldry, I. K., Balogh, M. L., Bower, R., Glazebrook, K., \& Nichol, R. C. 2004, in The New Cosmology: Conference on Strings and Cosmology, AIP Conf. Proc., 743106

Balogh, M. L., Baldry, I. K., Nichol, R., et al. 2004, ApJ, 615, L101

Bernardi, M., Sheth, R. K., Nichol, R. C., Schneider, D. P., \& Brinkmann, J. 2005, AJ, 129, 61

Blanton, M. R., Hogg, D. W., Bahcall, N. A., et al. 2003, ApJ, 592, 819

Bolejko, K., Krasiński, A., \& Hellaby, C. 2005, MNRAS, 362, 213

Croton, D. J., Farrar, G. R., Norberg, P., et al. 2005, MNRAS, 356, 1155

Dekel, A., \& Silk, J. 1986, ApJ, 303, 39

Dressler, A. 1980, ApJ, 236, 351

Einasto, J., Einasto, M., Hütsi, G., et al. 2003a, A\&A, 410, 425

Einasto, J., Hütsi, G., Einasto, M., et al. 2003b, A\&A, 405, 425

Einasto, J., Tago, E., Einasto, M., et al. 2005, A\&A, 439, 45

Fadda, D., Girardi, M., Giuricin, G., Mardirossian, F., \& Mezzetti, M. 1996, ApJ, 473,670

Fukugita, M., Ichikawa, T., Gunn, J. E., et al. 1996, AJ, 111, 1748

Goldberg, D. M., Jones, T. D., Hoyle, F., et al. 2005, ApJ, 621, 643

Gómez, P. L., Nichol, R. C., Miller, C. J., et al. 2003, ApJ, 584, 210

Gottlöber, S., Łokas, E. L., Klypin, A., \& Hoffman, Y. 2003, MNRAS, 344, 715

Grogin, N. A., \& Geller, M. J. 1999, AJ, 118, 2561

Grogin, N. A., \& Geller, M. J. 2000, AJ, 119, 32

Gunn, J. E., Carr, M., Rockosi, C., et al. 1998, AJ, 116, 3040

Hoeft, M., Yepes, G., Gottloeber, S., \& Springel, V. 2005, ArXiv Astrophysics e-prints

Hogg, D. W., Blanton, M. R., Eisenstein, D. J., et al. 2003, ApJ, 585, L5

Hoyle, F., \& Vogeley, M. S. 2004, ApJ, 607, 751

Hoyle, F., Rojas, R. R., Vogeley, M. S., \& Brinkmann, J. 2005a, ApJ, 620, 618

Hoyle, F., Vogeley, M. S., \& Rojas, R. R. 2005b, Amer. Astron. Soc. Meeting Abstracts, 206, 10.02

Kewley, L. J., Dopita, M. A., Sutherland, R. S., Heisler, C. A., \& Trevena, J. 2001, ApJ, 556, 121

Lindner, U., Einasto, J., Einasto, M., et al. 1995, A\&A, 301, 329

Lindner, U., Einasto, M., Einasto, J., et al. 1996, A\&A, 314, 1

Menci, N., Fontana, A., Giallongo, E., \& Salimbeni, S. 2005, ApJ, 632, 49

Olson, D. W., \& Silk, J. 1979, ApJ, 233, 395

Patiri, S. G., Prada, F., Holtzman, J., Klypin, A., \& Betancort-Rijo, J. 2006, MNRAS, 372, 1710

Peebles, P. J. E. 2001, ApJ, 557, 495

Pimbblet, K. A. 2005, MNRAS, 358, 256

Popescu, C. C., Hopp, U., Hagen, H. J., \& Elsaesser, H. 1996, A\&AS, 116, 43

Popescu, C. C., Hopp, U., \& Elsaesser, H. 1997, A\&A, 325, 881

Roberts, S., Davies, J., Sabatini, S., et al. 2004, MNRAS, 352, 478

Rojas, R. R., Vogeley, M. S., Hoyle, F., \& Brinkmann, J. 2004, ApJ, 617, 50

Sabatini, S., Davies, J., Scaramella, R., et al. 2003, MNRAS, 341, 981

Sabatini, S., Davies, J., van Driel, W., et al. 2005, MNRAS, 357, 819

Silk, J. 2004, in The New Cosmology: Conference on Strings and Cosmology, AIP Conf. Proc., 74333

Stoughton, C., Lupton, R. H., Bernardi, M., et al. 2002, AJ, 123, 485

Strateva, I., Ivezić, Ž., Knapp, G. R., et al. 2001, AJ, 122, 1861

Tanaka, M., Goto, T., Okamura, S., Shimasaku, K., \& Brinkmann, J. 2004, AJ, 128,2677

Wilman, D. J., Balogh, M. L., Bower, R. G., et al. 2005, MNRAS, 358, 88

York, D. G., Adelman, J., Anderson, J. E., et al. 2000, AJ, 120, 1579 
G. Sorrentino et al.: Galaxy properties from voids to clusters, Online Material p 1

\section{Online Material}




\section{Appendix A: The environment smoothing scale}

In this appendix, we will examine how changing the environment smoothing scale with which we define the local galaxy density affects our results. To this aim, we compare the $(u-r)$ color distribution for the galaxies in our sample in different environments, using three different smoothing scales: $2.5 \mathrm{Mpc}, 5 \mathrm{Mpc}$ (the reference value adopted in this paper), and $10 \mathrm{Mpc}$. These values are similar to those adopted by Einasto et al. (2005). We define the underdense environment $N<4$ (10th percentile) and the overdense environment for $N>59$ (90th percentile), as in the paper. In the top panel of Fig. A.1 we report the central panel of Fig. 2. In the middle panel we compare the $(u-r)$ color distribution for underdense (left) and overdense (right) environments, using three different smoothing scale: $2.5 \mathrm{Mpc}, 5 \mathrm{Mpc}$ (the reference value), and $10 \mathrm{Mpc}$. Finally, in the bottom panel we quantify the differences in the previous plots, that are the differences of the median $(u-r)$ color distribution for 2.5 and $10 \mathrm{Mpc}$ with respect to the reference value of of the $(u-r)$ color distribution for $5 \mathrm{Mpc}$ adopted in the paper. These differences are plotted both for the underdense (left panel) and overdense (right panel) environments, with values $(u-r)-(u-r)_{\text {ref }}<0.03$.

The previous analysis demonstrates that the adopted value of smoothing scale $(5 \mathrm{Mpc})$ is a robust representation and a good probe of both the underdense and overdense regions of the survey volume.
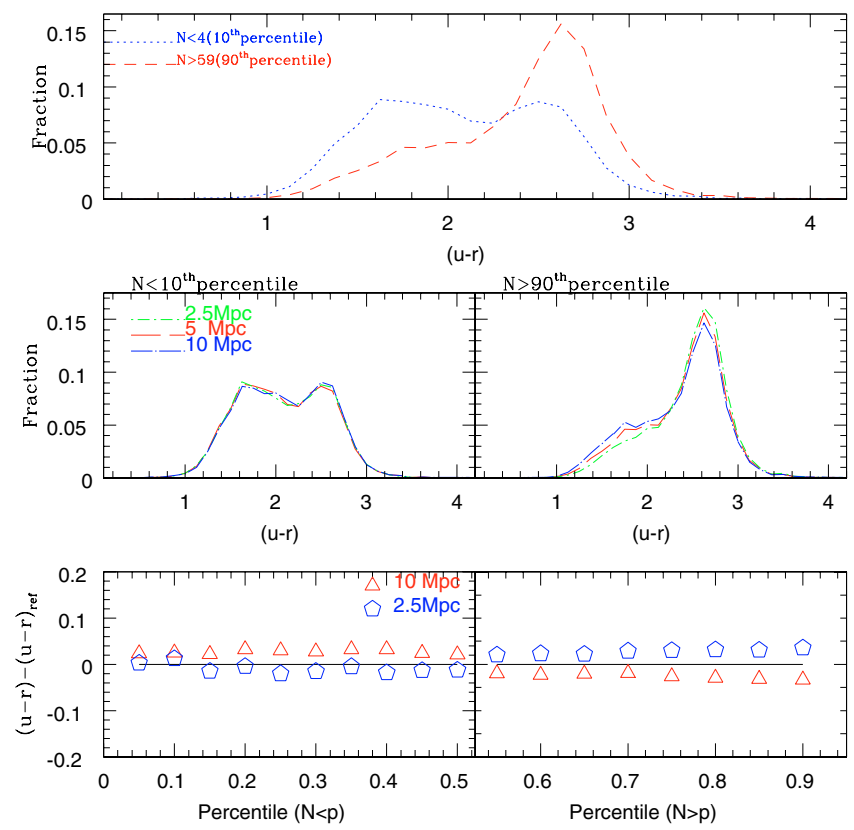

Fig. A.1. Top panel: the $(u-r)$ color distribution for a smoothing scale value of $5 \mathrm{Mpc}$ (the reference value), in the underdense and overdense environments. Middle panel: as in the top panel, for three different values of smoothing scale: $2.5 \mathrm{Mpc}, 5 \mathrm{Mpc}$, and $10 \mathrm{Mpc}$ (left panel: underdense environments; right panel:overdense environments). Bottom panel: differences in the $(u-r)$ color distribution respect to the reference values, for different environments (left panel: underdense; right panel: overdense). 